\title{
El desafío de articular una \\ experiencia extensionista a la currícula del taller de diseño
}

\author{
Martín Bomrad \\ martinbomrad@hotmail.com
}

Horacio Gorodischer

gorodischer@fadu.unl.edu.ar

Silvia Torres Luyo

storresluyo@yahoo.com.ar

\author{
Mariana Oliva \\ mariusa76@hotmail.com \\ Docentes de la Facultad de \\ Arquitectura, Diseño y Urbanismo. \\ Universidad Nacional del Litoral, \\ Argentina
}

Integración de la docencia y la extensión /

Intervenciones

RECEPCIÓN: 24/06/16

ACEPTACIÓN FINAL: 10/10/16

\section{Resumen}

En el taller de Diseño III de la carrera Licenciatura en Diseño de la Comunicación Visual, en la Facultad de Arquitectura, Diseño y Urbanismo de la Universidad Nacional del Litoral, el cuerpo docente trabajó los contenidos curriculares de la materia adaptándolos y aplicándolos a una situación real y concreta en el marco de un Proyecto de Extensión de Cátedra. El desafío que se presentaba era doble: por un lado, poder cumplir con el proceso de aprendizaje habitual, respondiendo a los contenidos y consignas fijadas en el programa de la materia, y por el otro, atender a las especificidades del caso con el objetivo de satisfacer las expectativas y necesidades reales del comitente. El proceso del proyecto y los alcances obtenidos demostraron que cumplir con el desafío era una tarea posible.

Palabras-clave

- Docencia y extensión

- Taller de diseño

- Derechos de expresión

- Programas visuales

- Revistas sociales

\section{Resumo}

Na oficina de Desing III do curso de Bacharelado em Desing da Comunicação Visual, na Faculdade de Arquitetura, Desing e Urbanismo da Universidad Nacional del Litoral, o corpo docente, juntamente com os alunos desse nível, trabalharam os conteúdos curriculares da disciplina adaptándo-os e aplicando-os a uma situação real e concreta no marco de um Projeto de Extensão Universitária. O desafio apresentado era duplo: por um lado, poder cumprir com o processo de aprendizado habitual, respondendo aos conteúdos e objetivos estabelecidos no programa de ensino da disciplina e, por outro, atender às especificidades do caso, com o objetivo de satisfazer as expectativas e necessidades reais do comitente. O processo do projeto e os alcances obtidos demonstraram que cumprir com o desafio era uma tarefa possível.

Palavras-chave

- Docência e extensão

- Oficina de design gráfico

- Direito de expressão

- Programas visuais

- Revistas sociais

\section{Para citación de este artículo}

Bomrad, M.; Gorodischer, H.; Torres Luyo, S.; Oliva, M.

(2016). El desafío de articular una experiencia extensionista a la currícula del taller de diseño. En Revista $+E$ versión digital, (6), pp. 422-429. Santa Fe, Argentina: Ediciones UNL. 


\section{La práctica extensionista universitaria dentro del taller de diseño}

La extensión es una función esencial de la Universidad Nacional del Litoral (UNL). Dicha tarea se centra en la búsqueda de soluciones a los problemas de la región y en mejorar la calidad de vida de la población. Esto lo hace mediante el fortalecimiento de espacios de articulación, la generación de acciones colaborativas en estrecha interacción con la sociedad en su conjunto y la socialización del conocimiento generado y procurando concretar acciones transformadoras.

En los Proyectos de Extensión participan docentes, pasantes y estudiantes, como así también los beneficiarios directos. Esta participación se da tanto en la planificación como en la ejecución de los proyectos, conformando un entramado de relaciones que pretende construir una mirada que exceda los esfuerzos individuales y descontextualizados de la realidad que los rodea. Los talleres de diseño son asignaturas troncales en la carrera de la Licenciatura en Diseño de la Comunicación Visual de la Facultad de Arquitectura, Diseño y Urbanismo, y demandan al alumno la integración de los conocimientos que se imparten en las diferentes áreas del saber y que se dictan según su plan de estudio: de ciencias sociales, de tecnología y de diseño. Dentro de estas áreas las materias se plantean con un corte teórico o de índole proyectual. Los talleres de diseño transfieren este último modo de conocimiento.

El conocimiento proyectual, según Mazzeo y Romano (2007:57), "forma parte de un grupo de disciplinas que sólo pueden aprenderse en la práctica, aunque parezca paradójico, a diseñar se aprende diseñando".

Por lo tanto, el alumno debe hacer una transferencia de lo aprendido en las materias teóricas y en las de índole proyectual, para luego ponerlos en práctica en los talleres.

La práctica habitual de enseñanza en los talleres de diseño es planteada muchas veces como un simulacro de la realidad, mediante ejercicios que pretenden imitar algunas instancias del trabajo profesional. Sin embargo, estos ejercicios pueden tener muchas similitudes tanto como diferencias con la realidad, lo fundamental es que puedan ser adaptados pedagógicamente y apropiados para la enseñanza, favoreciendo y estimulando el interés de los alumnos.

Mazzeo y Romano (2007) sostienen que estas simulaciones de la realidad profesional que se dan en los talleres para plantear los contenidos curriculares, excluyen siempre alguna etapa del proceso proyectual profesional. Por ejemplo, si bien las nuevas tecnologías sirven para acortar la proximidad de la pieza diseñada por los alumnos con un objeto real, esta proximidad de la simulación a la realidad se da en su concreción, y no así en su uso, función y contextualización.

Ahora bien, la implementación de un Proyecto de Extensión en la práctica de enseñanza de los talleres de diseño nos plantea, en principio, el desafío para integrar un contexto real con los contenidos curriculares habituales. Y nos abre los siguientes interrogantes: ¿Puede la extensión universitaria hacer factible la posibilidad de dejar de proyectar sobre situaciones hipotéticas y simuladas, para desafiar un problema real y contextual, tratando de incluir todas las etapas de un proceso proyectual profesional, sin postergar los contenidos y el interés del alumno?

¿El trabajar con comitentes reales puede viabilizar una práctica reflexiva y crítica en todos los actores participantes, ayudando a salirse de la mirada pasiva que muchas veces paraliza la creatividad en la enseñanza proyectual?

Describir y reflexionar sobre este proceso, a partir de un caso concreto de extensión universitaria, implica responder estos interrogantes, para problematizar y repensar las prácticas de enseñanza y aprendizaje dentro del Taller.

\section{Introducción a las especificidades del caso concreto}

En la Argentina de los últimos años se han dado dos circunstancias relevantes que hacen a la problemática que se pretende abordar. Por un lado, la aprobación de la Ley de Protección Integral de los Derechos de Niños, Niñas y Adolescentes (26061), que sustenta el respeto pleno de los mismos derechos humanos básicos que se les reconoce a los adultos. El otro hecho importante fue la sanción de la nueva Ley de Medios (26522), que dio origen e instaló en la opinión pública la problemática y discusión del rol de los medios de comunicación.

En este contexto surgen diferentes sectores (asociaciones, cooperativas, ONG, comunidades de pueblos originarios, etc.) que, frente a la falta de espacios de comunicación, buscan generar un lugar para dar a conocer sus realidades, situaciones sociales, culturales, educativas, etc., para ser protagonistas de sus propias historias.

Estas dos cuestiones, ligadas a la idea del rol responsable que debe cumplir el Estado para que estas leyes se garanticen, nos definen el problema a abordar: la necesidad que manifiesta un sector de la sociedad santafesina (niños y jóvenes en situación de calle) de integrarse socialmente y satisfacer sus derechos a expresarse. Ante esta situación, desde el Taller de Diseño III recuperamos la concepción de María del Valle Ledesma (2010) respecto de plantear al diseño como disciplina que pueda generar una voz institucional para los que no la tienen y necesitan tenerla. Este pensamiento propone dar un salto a la acción en el ámbito de las problemáticas sociales, en un intento de superar las tradicionales concepciones utilitaristas, monetarias e individualistas que muchas veces dominan las escenas del diseño.

A raíz de estos intereses, en el año 2010 se organizó un encuentro de los docentes y alumnos del Taller III con la Asociación Civil Juanito Laguna de la ciudad de Santa Fe, que trabaja con niños y jóvenes en situación de vulnerabilidad social. En esa oportunidad 
—en la cual los alumnos hicieron un trabajo de campo para luego realizar un trabajo práctico- los responsables de la Asociación nos expresaron el anhelo por contar con un soporte de comunicación propio que sirva como medio para darse a conocer. Esta experiencia de realizar un trabajo práctico sobre un destinatario concreto y cercano, apoyada por el manifiesto interés del alumnado de la cátedra, cimentó las bases para que el grupo docente planteara un futuro trabajo bajo la modalidad de Proyecto de Extensión de Cátedra.

\section{Correspondencia entre el proyecto pedagógico y el de extensión}

La materia Taller de Diseño III, Cátedra Gorodischer, toma su nombre del arquitecto y diseñador Horacio F. Gorodischer, quien es el profesor titular. Esta denominación es tradición en las carreras proyectuales, Arquitectura y los diversos Diseño, para diferenciar las propuestas de cátedras que los alumnos pueden elegir para el cursado.

La materia se organiza bajo la modalidad de taller, con clases teóricas que apoyan y sirven de introducción a los problemas que se plantean a lo largo del cuatrimestre. El equipo docente se completa con un profesor adjunto y jefes de trabajos prácticos que guían al alumno en su aprendizaje.

La designación de taller tiene su origen en el espacio físico en el cual docentes y alumnos interactúan y desarrollan el proceso de enseñanza-aprendizaje. El origen, aunque no esté muy claro, se remonta a la edad media, donde maestros y discípulos compartían un espacio de trabajo, concepción que institucionalizada y con modificaciones siguió la Bauhaus, posteriormente la escuela de UIm, y actualmente es utilizada por diferentes cátedras en las carreras de diseño de las universidades argentinas.

Para abordar la experiencia extensionista con una materia de corte proyectual, se propuso como marco teórico los contenidos desarrollados a lo largo del cuatrimestre y basados en la bibliografía ${ }^{1}$ recomendada por la cátedra. En lo que respecta a las metodologías adoptada, se siguió un enfoque cualitativo y el proceso proyectual fue definido por etapas operativas.

Particularmente, el nivel III de los talleres de la cátedra plantea sus ejes en prácticas de enseñanzas proyectuales que involucren modos de visualización apropiados para estructuras narrativas complejas. Esto significa adquirir y perfeccionar habilidades conceptuales para proyectar programas visuales y para categorizar y jerarquizar sistemas de numerosas variables. Se ponen, entonces, en juego los saberes producidos para entrenarse en la capacidad de realizar dispositivos complejos, productores de sentidos explícitos y portadores de informaciones múltiples. Dispositivos que deben definir por sí mismos sus modalidades de uso, recorrido, comunicación e interacción con el usuario, entendido éste como un sujeto individual y a la vez, colectivo.

Los contenidos y trabajos prácticos o proyectos tienen una estructura que plantea diferentes niveles de profundidad y complejidad a medida que avanzan. La resignificación en nuevos trabajos es fundamental para alcanzar los objetivos; y el último proyecto del Taller consiste en diseñar una revista.

Desde la cátedra, detectamos la correspondencia entre las necesidades comunicacionales de la Asociación y los contenidos fundamentales del Taller, en particular con aquellos que se vinculan a la elaboración de estrategias orientadas a programas visuales de identidad, de información y editoriales, ya que durante el segundo cuatrimestre la planificación curricular aborda estas problemáticas a través de la resolución de dos piezas concretas: un manual de pautas para una revista, y el desarrollo de dicha revista a partir de la hipótesis proyectual que el manual previo supone.

Que el objeto resultante del proceso de diseño fuera un material editorial impreso posibilitó el intercambio de saberes entre las dos partes involucradas, entre los que cabe señalar la posibilidad de incorporar conocimientos de un oficio para los estudiantes del taller, dado que la Asociación cuenta con un taller de imprenta en torno al cual giran la mayoría de sus actividades. Dentro de las instalaciones, este taller funciona como herramienta de aprendizaje de oficios, dirigido a la integración social de los jóvenes que participan, además de servir como medio para autosustentarse. Si bien algunas de las consignas debieron ser reformuladas, encuadrándolas dentro del formato "revista social" para poder responder a las necesidades específicas de la Asociación, muchas de las características esenciales del proyecto pedagógico se mantuvieron para asegurar que los alumnos cumplieran con el proceso de aprendizaje al igual que todos los años.

Este replanteo posibilitó una organización del trabajo en forma superadora, tanto para los jóvenes y educadores de la Asociación —dado que participaron del proceso de diseño— como para los alumnos y docentes de la cátedra, que debieron enfrentarse ante una necesidad real, conflictiva y con sus propias limitaciones para responder a la consigna ateniéndose a las peculiaridades del caso. El trabajo exigió cooperación, pensamiento colectivo y articulación de distintos saberes; cada uno de los participantes debió primero poseer una idea del conjunto del trabajo, tornándose en consecuencia, más reflexivo y efectivo para el desempeño de sus tareas específicas. 


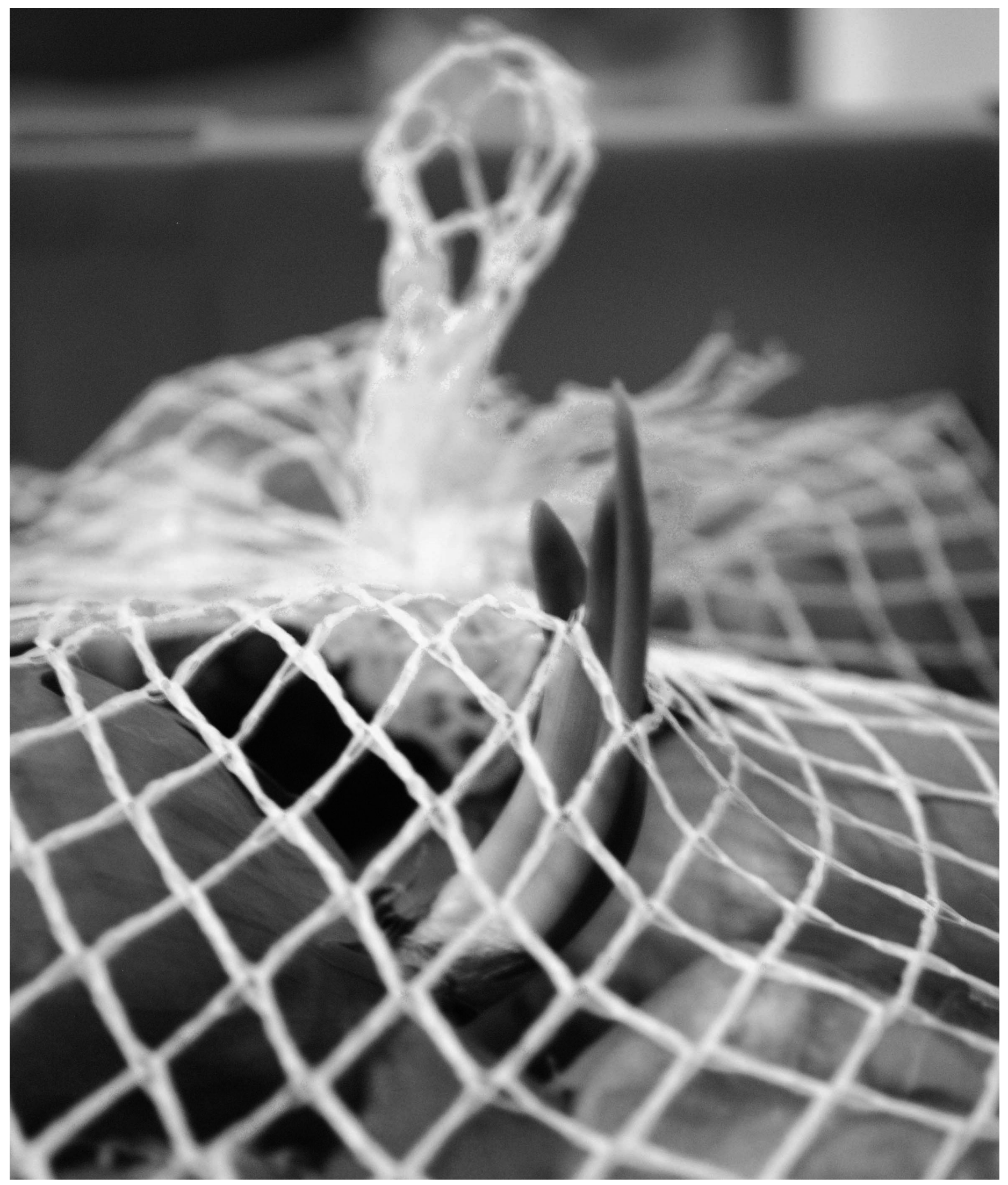

(c) Laura Hormaeche 


\section{Desarrollo y reflexiones del Proyecto de Extensión}

Las disciplinas proyectuales tienen su esencia en su proceso de generación, y esto hace a su coherencia. Para Mazzeo y Romano (2007), dicho proceso puede ser explicito o implícito, ser consciente o no, pero siempre posee una secuencia que en la enseñanza es conveniente explicitar para poder orientarlo.

Esta secuencia no está constituida por etapas fijas, finitas, ni lineales. Sin embargo, para un proyecto que implica la participación de varios actores sociales (docentes, alumnos e integrantes de la Asociación), que está inserto en un proceso de aprendizaje, que depende de un tiempo -entre otras consideraciones-, es fundamental establecer un ordenamiento operativo en etapas para garantizar un nivel deseable de productividad.

La propuesta del proceso proyectual que plantean Mazzeo y Romano (2007) incluye etapas de información, de formulación, de desarrollo y por último una etapa de materialización y verificación del objeto diseñado.

Estas etapas en orden consecutivo de tiempo están atravesadas por una instancia de comunicación permanente del diseñador hacia terceros como con un dialogo consigo mismo.

El Proyecto de Extensión realizado incluyó la secuencia de estas etapas, implicando los contenidos y metodologías propios del Taller de Diseño III en un constante trabajo de articulación y adaptación a las necesidades del comitente real.

En la fase inicial del proyecto, en la etapa de información, se debe profundizar en los requerimientos surgidos del análisis de las necesidades del usuario/destinatario, de las condiciones del medio físico y cultural, de los determinantes propios de los sistemas de producción disponibles, de los antecedentes del tema y cómo estos han dado respuestas desde el diseño a problemas afines. Fue entonces necesario realizar un análisis para poder conocer la situación de la Asociación Civil Juanito Laguna, la problemática que trata, su funcionamiento y recursos, sus acciones comunicacionales e identidad. Esto se logró a través de una sucesión de encuentros, con entrevistas no estructuradas a partir del diálogo, con exposiciones y trabajos de campo entre el taller, inmerso en un ámbito universitario, y la Asociación con su trabajo en la sociedad civil.

Además, se seleccionó un material bibliográfico específico sobre casos análogos orientado a soluciones de diseño en ámbitos de compromiso social y político que sirvieron como antecedentes del tema, y en paralelo, se dictaron clases teóricas sobre diseño de identidad y recursos editoriales.

Por último se organizó una jornada especial donde los alumnos del Taller III y los jóvenes y encargados de la Asociación realizaron un análisis - sincrónico y diacrónico- de revistas de carácter social y lo visualizaron a través de una serie de paneles. En disciplinas como el diseño, la metodología de recuperación de trabajos profesionales como referentes permite la adquisición y transferencia de los saberes de experiencias previas y la confirmación —o no- de supuestos disciplinares aún no verificados en el trabajo en curso.

Esta etapa enmarcada en un Proyecto de Extensión nos posibilitó conocer una realidad e iniciar desde el taller el intercambio de saberes, el conocimiento de los recursos y limitaciones mutuas, de modo de poder empezar a pensar en el siguiente paso.

La etapa de formulación es donde se clarifican las ideas, para aplicarlas sobre las futuras piezas visuales.

Por lo tanto, consistió en el planteo de las piezas gráficas a diseñar por parte de los alumnos: un manual de pautas para la revista de la Asociación y la revista en sí. Proyectar sobre estas dos piezas en particular posibilitó explicitar los conceptos de programa visual, sistema e identidad, que son de fundamental relevancia dentro de los contenidos curriculares del Taller de Diseño III, siguiendo, al igual que en otros años, las teorías y los autores que nos sirven de apoyo para entender estos conceptos.

Con relación a la primera pieza, entendemos al manual de pautas como un programa visual que está constituido por determinados elementos y determinadas reglas de combinación.

En un programa diseñado, Paul Gredinger asevera:

"estos y aquellos son sus elementos. Y esto es lo que puedo hacer con ellos. Resultado: toda una serie de soluciones. Lo importante no es que el resultado sea éste o aquel; lo importante es que la forma se cree - tenga que crearse - obedeciendo a un orden o fórmula". (Gerstner, 1979:9)

Por lo tanto, es en el diseño de la fórmula, por ejemplo, en un manual, las pautas planteadas, y no en el diseño de la forma, los recursos editoriales e imágenes de la revista, donde existe la creación y por consiguiente el objetivo de la configuración del programa visual.

Por otra parte, para abordar una revista aplicamos la definición de sistema de Karl Gerstner (1979), en cuanto ésta es más que una simple suma de partes — cubierta, sumario, notas, etc.—o de recursos verbales y visuales. Sino que son sus partes, sus funciones individuales, sus recursos y las relaciones entre ellos los que la configuran como tal. Consecuentemente, dichas partes y recursos también formarán otros sistemas y subsistemas.

Así, una revista como sistema se construirá a partir de variables y constantes en el diseño de sus partes y recursos que deberán ser jerarquizadas: ponderación en cuanto a una definición y acentuación de importancia, y categorizadas: diferenciación en cuanto a tipología y función, para una sistematización en su aplicación a lo largo de la construcción de las revistas con sus particularidades.

Esta forma de concebir a las revistas conlleva intrínsecamente a la construcción de una identidad determinada si se sostiene en el tiempo. Aunque no es intención de este texto indagar en profundidad sobre el concepto de identidad, cuando nos referimos 


\title{
66
}

\author{
la realización de este proyecto contribuyó \\ a la concientización y al reconocimiento \\ de los alcances, las incumbencias y los \\ beneficios de la inserción de la disciplina \\ en terrenos sociales pocos desarrollados
}

a éste lo hacemos desde el enfoque de A. Semprini (1995), quien retoma los textos de Algirdas Greimas y colaboradores, lo que es conocido como La Escuela de París. Este enfoque postula que la identidad es la forma en la que algo se hace visible y se materializa en los discursos que los actores sociales cruzan entre sí, proponiendo para ésta un método de creación, gestión y análisis, a partir de tres niveles que posibilitan construir sentido: nivel axiológico, narrativo y superficial.

Desde este planteo, diseñar una revista significa construir una visualización apropiada (nivel superficial) para una estructura narrativa compleja (nivel narrativo), productora de sentidos explícitos y portadora de información múltiple (nivel axiológico). $Y$ esto constituye un gran desafío, que presupone enfrentar problemas compositivos, tipográficos, integración entre imagen y texto, mecánica de recorrido y ritmos, de identidad y producción de sentidos, entre otros.

Este proceso, a partir de estas dos piezas gráficas en particular, baja estos conceptos. Al ser aplicado a la resolución de un caso concreto, demostró ser operativo para que el alumno promueva un pensamiento crítico y reflexivo sobre su propia producción. El ida y vuelta que propone la programación de un manual de pautas y su aplicación al soporte revista hace evidente su concepción de sistema transmisor de identidad y factible comprender y corroborar su funcionamiento.

Concluida la etapa de formulación de las ideas conceptuales y formales sobre la Asociación, el alumno, con el apoyo docente, fue desarrollando el diseño del manual de pautas y consecuentemente la revista con su particular identidad visual. En esta etapa de desarrollo, las propuestas de diseño se van materializando primero bajo la forma de bocetos hasta llegar al arte final. Mazzeo y Romano (2007), recuperando el «Postulado de la externalización» de Jerome Bruner, plantean que la materialización pone las ideas del alumno a su alcance y al alcance del docente, lo que viabiliza la producción de reflexiones operativas sobre lo producido. "La externalización produce un registro de nuestros esfuerzos mentales, un registro que está fuera de nosotros más que estar vagamente en la memoria. Es algo parecido a producir un borrador, un esquema general, un simulacro" (Bruner, 1993, citado por Mazzeo y Romano, 2007:60).

Durante esta etapa de desarrollo, que comienza con un alto grado de generalidades, el alumno proyecta sus propuestas de diseño, en un dialogo con el docente, con los otros alumnos y consigo mismo. Lo hace a través de diferentes instancias y modos de correcciones, tales como enchinchadas, exposiciones grupales o individuales, hasta definir lo que será su entrega final a ser evaluada.

Como caso especial al Proyecto de Extensión y cierre de esta etapa, se realizó una jornada de exposición de las propuestas de diseños que permitió a los alumnos del taller presentar las diferentes identidades a los directivos de la Asociación. La exposición cumplió con las expectativas de los responsables, quienes participaron junto a los docentes del taller en la selección de tres manuales. Estos tres manuales fueron puestos a prueba a través de un 
workshop como una instancia simulada de verificación curricular y autoevaluación que finalizó con la elección del manual de pautas definitivo a ser utilizado por la Asociación.

Los objetivos de la consigna, tanto desde el punto de vista de los intereses de la cátedra como desde las expectativas de la Asociación, fueron superados ampliamente, dado que los diseños lograron poner en palabras e imágenes varias propuestas de identidad, todas pensadas y construidas para visualizar el discurso de la Asociación. A su vez, comparativamente con años anteriores, detectamos un compromiso más activo por parte de los alumnos, consecuencia de la generación de soluciones en respuesta a un problema real, en el que fue necesario adaptarse a las situaciones del contexto y a las particularidades del comitente.

La mecánica de trabajo, sobre la base de sucesivos encuentros como instancias de comunicación con la Asociación, permitió introducir a los alumnos en la experiencia de la transmisión de conocimientos, conceptos e imágenes de producción propias a un comitente real y los enfrentó a la instancia de la presentación, donde además de verse obligados a preparar la exposición tuvieron la posibilidad de probar y recibir una devolución de sus propuestas, lo cual produjo un interesante modo de trabajo sustentado en la retroalimentación de ambas partes.

Hasta esas instancias, todos los alumnos del taller trabajaron a nivel de arte final y presentaron los diseños del manual de pautas y su respectiva revista como último proyecto del año con el fin de cumplir con los objetivos curriculares.

En paralelo, un grupo reducido de alumnos trabajó en el diseño y la maquetación del primer ejemplar de la revista de la Asociación Civil Juanito Laguna, Ilamada Malabares. Este ejemplar fue elaborado poniendo en uso el manual de pautas seleccionado una vez verificado su funcionamiento.

Para obtener los materiales a ser utilizados en la revista se definieron los contenidos: textos reales de autores, ilustraciones, tipografías, esquemas y producciones fotográficas. Esto se realizó a través de un trabajo en conjunto entre docentes, alumnos e integrantes de la Asociación. Sobre dichos materiales se tuvieron en cuenta los aspectos legales y derechos de autor, que no surgen rigurosamente en la práctica habitual de la enseñanza dentro del taller.

Una vez logrado el original de la revista, se trabajó en los talleres gráficos de la Asociación para la impresión en sistema ófset del primer ejemplar y se obtuvo así una tirada masiva. De esta manera, el Proyecto de Extensión posibilitó abordar realmente la etapa de materialización del proceso proyectual y, al mismo tiempo, los alumnos lograron tener un contacto real con la práctica profesional en lo que se refiere a la impresión de piezas gráficas.

Las etapas de materialización y verificación, por estar incluidas dentro de un Proyecto de Extensión, pudieron concretarse y dieron como resultado:

1) Un manual de pautas, ideado exclusivamente para la Asociación y pensado como herramienta guía, cuya finalidad es organizar las estrategias comunicacionales y la identidad editorial de la revista social que representará a la Asociación durante un extenso período de tiempo. La existencia de este soporte admite la edición continua de la revista dado que permite su diagramación y edición por parte de los integrantes de la Asociación de manera independiente y autogestionaria.

2) El diseño, la maquetación, la impresión y la distribución de la revista Malabares $n^{\circ} 1$, revista de la Asociación Civil Juanito Laguna, como resultado y puesta en acto del manual de pautas anteriormente citado. Esta revista, como medio de comunicación inserto en el ámbito urbano, se convierte así en el soporte gráfico transmisor de la identidad construida, transformándola en portavoz de la Asociación y referente visual de la problemática social representada.

Al finalizar el año lectivo se realizó una exhibición del proyecto ${ }^{2}$ y se presentó la revista Malabares $n^{\circ} 1$ ante la comunidad en la Facultad. Luego, este primer ejemplar fue presentado por parte de la Asociación a la sociedad santafesina en general.

\section{Resultados obtenidos}

En lo disciplinar, la realización de este proyecto contribuyó a la concientización y al reconocimiento de los alcances, las incumbencias y los beneficios de la inserción de la disciplina en terrenos sociales pocos desarrollados, además de ampliar la formación de los futuros profesionales del diseño en comunicación visual.

En el ámbito de lo académico, en lo que atañe al equipo docente, el proyecto nos condujo a un replanteo de las actividades realizadas en el taller y amplió nuestro horizonte a través de nuevas relaciones y contactos generados con otras cátedras del país que realizan prácticas similares. En el alumnado, se logró pensar sobre 
las acciones comunicacionales que produce un diseñador a través de una mirada crítica. Los alumnos pudieron tomar conciencia de su rol social como factor de transformación y desarrollo del entorno donde viven. Tanto los alumnos como los docentes involucrados en el proyecto tuvieron la posibilidad de generar un trabajo comprometido, llevado a cabo a través de una práctica reflexiva, que deja las bases para nuevas iniciativas en el campo de la extensión para las disciplinas proyectuales.

Finalmente, en el ámbito de lo social, la propuesta contribuyó a los planes de reinserción social de los jóvenes incluidos en el Proyecto a partir de la generación de un medio impreso que satisfaga sus derechos y necesidades de expresarse, de aprender un oficio por medio de la práctica y de relacionarse con los participantes del complejo proceso que demanda la publicación de una revista.

\section{Conclusión}

Desde nuestro rol de educadores pudimos ratificar las hipótesis planteadas de que es posible tomar una problemática concreta y hacerla funcionar como argumento para actualizar el dictado de contenidos curriculares regulares del taller de un modo específico, sin obstaculizar el proceso de aprendizaje sino más bien todo lo contrario, fomentándolo gracias a la generación en el alumno de una actitud receptiva con el Proyecto y comprometida con el comitente, haciéndolo sentir partícipe en una acción transformadora.

En segundo lugar, como profesores universitarios, logramos no solo satisfacer los objetivos del Programa de Extensión sino también explotar la coyuntura que nos ofrece la Universidad a los fines de una mejor internalización de los contenidos de nuestra materia en particular y la puesta en valor de la disciplina en general en cuanto herramienta de cambio social.

Por último, y simplemente en calidad de ciudadanos pertenecientes a la comunidad de Santa Fe, conseguimos participar entre todos y desde diferentes funciones en la resolución de un caso de responsabilidad social que atañe a los derechos civiles (el derecho de expresión) de una minoría, en una dimensión operativa. El manual de pautas obtenido y el diseño y la impresión del primer ejemplar de la revista Malabares actúan en este sentido como herramientas nodales para la comunicación institucional de la Asociación Juanito Laguna, responden de modo pertinente y efectivo a la necesidad originalmente detectada y contribuyen, en consecuencia, al desarrollo sostenible de esta Asociación.

\section{C6}

la propuesta contribuyó a los

planes de reinserción social de los

jóvenes incluidos en el Proyecto

a partir de la generación de un medio

impreso que satisfaga sus derechos

y necesidades de expresarse

\section{Referencias bibliográficas}

Gerstner, K. (1979). Diseñar programas. Barcelona: Gustavo Gili.

Gorodischer, H. (2004). Taller Vertical de diseño II, III y IV. Polis (Edición especial. Décimo aniversario de la carrera de diseño), 63. Santa Fe: FADU-UNL.

Ledesma, M. (2010). El diseño gráfico una voz pública. De la comunicación visual en la era del individualismo. Buenos Aires: Wolkowicz editores.

Mazzeo, C. y Romano, A. (2007). La enseñanza de las disciplinas proyectuales. Hacia la construcción de una didáctica para la enseñanza superior. Buenos Aires. Nobuko.

Semprini, A. (1995). El marketing de la marca: una aproximación semiótica. BarceIona: Paidós Ibérica. 